\title{
Chemotherapie mit pegyliertem liposomalen Doxorubicin in Kombination mit PUVA und Interferon-alpha bei Mycosis fungoides Stadium IIB
}

K. Mebes

P. von den Driesch

\section{Chemotherapy with Pegylated Liposomal Doxorubicin (PLD) in Combination with PUVA and Interferon-Alpha in Stage IIB Mycosis Fungoides (MF)}

\section{Zusammenfassung}

Die Mycosis fungoides (MF) ist eine der häufigsten Formen des kutanen T-Zell-Lymphoms. Bei fortgeschrittenen Stadien der MF ist die mittlere Lebenserwartung relativ kurz. Derzeit besteht kein standardisiertes oder definitives Therapieprotokoll. Ein 63-jähriger Patient mit rasch progressiver MF im Tumorstadium sprach auf die Therapie mit pegyliertem liposomalen Doxorubicin (PLD) in Kombination mit Interferon-alpha und PUVA an. Es wurden keine schweren Nebenwirkungen oder Infektionen beobachtet. Pegylierte Liposome sind stabile Trägermoleküle mit verlängerter Plasmazirkulation, die Doxorubicin selektiv im Tumorgewebe anreichern und somit weniger toxisch sind als freies Doxorubicin. Freies Doxorubicin wurde in vielen Therapieprotokollen zur Behandlung von Non-Hodgkin-Lymphomen eingesetzt. PLD ist erwiesenermaßen wirksam und sicher bei der Behandlung des rezidivierenden und therapieresistenten kutanen T-Zell-Lymphoms. Die aktuellen Daten empfehlen, PLD als Monotherapie einmal monatlich in einer Dosis von $20 \mathrm{mg} / \mathrm{m}^{2}$ anzuwenden. Unser Fall demonstriert, dass PLD bei Mycosis fungoides auch in Kombination mit anderen Therapien eingesetzt werden kann. Dies sollte in weiteren Studien untersucht werden.

\section{Abstract}

Mycosis fungoides (MF) is a common form of cutaneous T-cell lymphoma. Advanced stage MF is associated with a relatively short median survival. Currently there is no standard or definite curative treatment regimen. A 63 year-old man with rapid disease progression of tumour stage MF (T3, stage IIB) showed clinical response to pegylated liposomal doxorubicin (PLD) in combination with interferon-alpha and PUVA-treatment. No severe side-effects or infectious complications have been observed. Pegylated liposomes are stable, long-circulating carriers useful for delivering doxorubicin to tumour sites with a lower toxicity than the free drug. Free doxorubicin is used in several treatment protocols for Non-Hodgkin's lymphoma. It was shown that PLD was effective and safe in the treatment of relapsing or recalcitrant cutaneous T-cell lymphoma. Current data support the use of PLD as monochemotherapy given once per month at a dose of $20 \mathrm{mg} / \mathrm{m}^{2}$. Our case demonstrates that PLD can be used in combination regimens with other established mycosis fungoides therapies. This should be investigated in further studies.

\section{Einleitung}

Die Mycosis fungoides (MF) ist eine der häufigsten Formen kutaner T-Zell-Lymphome. Das Krankheitsbild ist im frühen Stadium durch ekzemartige Hautveränderungen und Plaques, im fortge- schrittenen Stadium durch Tumoren mit teilweise viszeraler Beteiligung gekennzeichnet. Im fortgeschrittenen Stadium ist die mittlere Lebenserwartung herabgesetzt und es gibt bislang keine kurative Therapieoption.

Institutsangaben

Klinik für Dermatologie und Allergologie, Zentrum für Hautkrankheiten, Klinikum Stuttgart (Direktor: Prof. Dr. P. von den Driesch)

Widmung

Prof. Dr. med. O.P. Hornstein zum 80. Geburtstag gewidmet

Korrespondenzadresse

Prof. Dr. Peter von den Driesch · Klinik für Dermatologie und Allergologie · Klinikum Stuttgart .

Prießnitzweg 24· 70374 Stuttgart·E-mail: pdriesch@kbc-intern.de

Bibliografie

Akt Dermatol 2006; 32: 322-325 @ Georg Thieme Verlag KG Stuttgart · New York

DOI 10.1055/s-2006-925078 · ISSN 0340-2541 
PUVA-Therapie und PUVA-Therapie in Kombination mit Interferon-alpha sind wirksame Behandlungen besonders in frühen Stadien der MF. Bei Rezidiven, Versagen der bisherigen Therapie oder rascher Progression nach Diagnosesicherung ist die Chemotherapie mit pegyliertem liposomalen Doxorubicin (PLD) eine sehr wirksame, nebenwirkungsarme Therapiealternative.

\section{Kasuistik}

\section{Anamnese}

Seit über sechs Jahren leidet der 63-jährige Patient an rezidivierenden „Ekzemen“ unterschiedlicher Ausprägung an wechselnden Hautarealen. Im Verlauf des vergangenen Jahres kam es zu einer schnellen Ausdehnung und Persistenz der Herde mit zunehmender Erhabenheit einzelner Stellen und der Bildung von Knoten. Zusätzlich bestand starker Juckreiz.

Eine im Jahr 2002 extern durchgeführte Biopsie ergab das Vorliegen einer lichenoiden, lymphohistiozytären exsudativen Entzündung der Dermis mit Epidermotropismus. Eine MF konnte nicht eindeutig nachgewiesen werden.

Bisher angewandte antiekzematöse Externa und Creme-PUVA erbrachten keine Besserung.

\section{Befund}

Bis zu $7 \mathrm{~cm}$ durchmessende blassrot bis rot-bräunliche, scharf begrenzte, teils konfluierende, schuppende, teils exsudative Plaques und Knoten, hauptsächlich an Kinn/Unterlippe, Oberarminnenseiten, am seitlichen Rumpf, Gesäß und Leisten beidseits. Ca. $7 \mathrm{~cm}$ messender indurierter Weichteiltumor links gluteal. Multiple Nävuszellnävi, vor allem am Rücken (Abb.1 -2).

\section{Histologie}

Wechselnd Hyperparakeratose, schmalgliedrige Akanthose der Epidermis, über die gesamte Läsionsbreite regelmäßig hochgradig atypische lymphozytäre Akkumulationen in der Epidermis im Sinne von Pautrier'schen Mikroabszessen. Subepithelial sehr dichtes, lymphozytär betontes Infiltrat mit hyperchromatischen entrundeten Zellkernen, dazwischen einzelne atypische Mitosen. Passend zu Mycosis fungoides.

\section{Labor}

Pathologisch verändert waren:

Initial:

CRP 3,3 mg/dl; Hb 13,0 g/dl, Erythrozyten $4 \times 10$ Mio./ $\mu$, Thrombozyten $126000 / \mu l$, IgE gesamt $829 \mathrm{kU} / \mathrm{l}$

Im Verlauf nach 4 Zyklen Chemotherapie mit PLD:

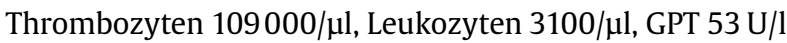

Nach 7 Zyklen Chemotherapie mit PLD:

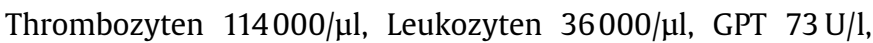
GOT $49 \mathrm{U} / \mathrm{l}$, GGT $79 \mathrm{U} / \mathrm{l}$

Normwerte für restliches Blutbild, Leber- und Nierenwerte, Elektrolyte, Sézary-Zellen unter $3 \%$.

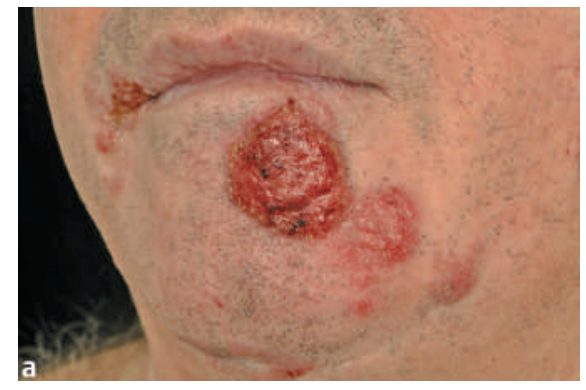

Abb. 1 a-c Befund eines Patienten mit Tumorstadium einer Mycosis fungoides

(a) Kinn, (b) Flanke, (c) Stamm
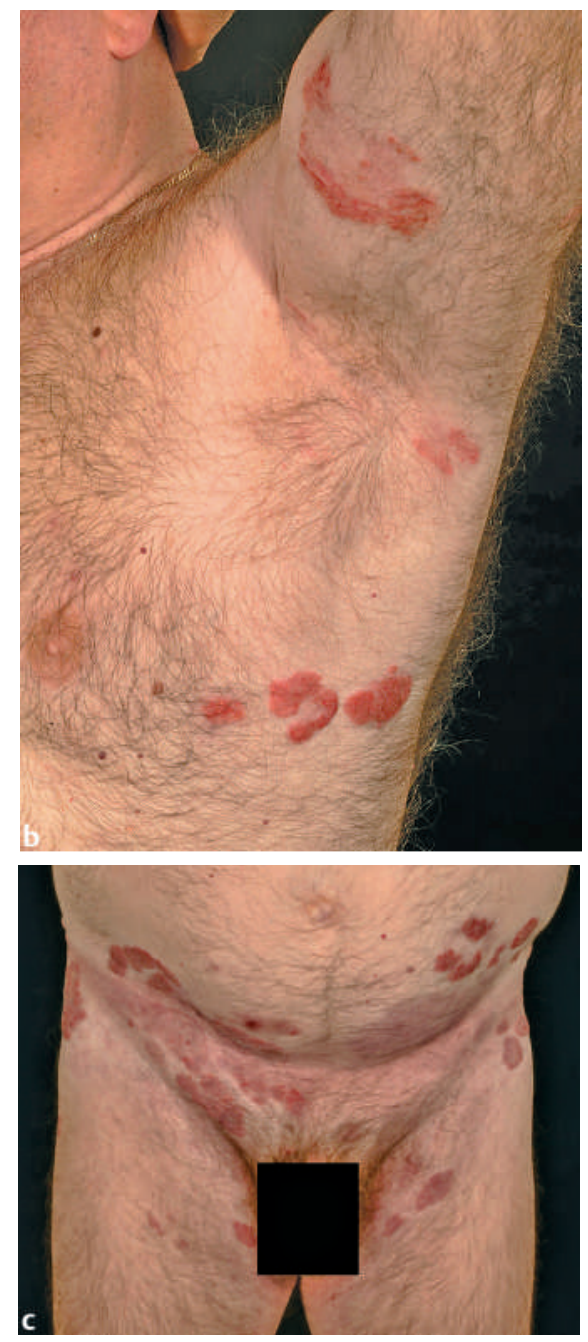

\section{Apparative Diagnostik}

EKG (vor Therapie und bei jedem 2. Zyklus): NFSR, LT, keine spezifischen ERBS.

Echokardiographie (vor Therapie und bei jeden 3. Zyklus): Regelrecht. Keine Einschränkung der LV-Funktion.

PET-CT (vor Therapie):

FDG-Mehrspeicherung kutan prämandibulär links und Gesäß links, vereinbar mit Manifestationen des kutanen Lymphoms.

Lymphknotensonographie (nach 7 Zyklen PLD): ohne pathologischen Befund.

CT-Thorax und Abdomen (nach 7 Zyklen PLD): ohne pathologischen Befund. 


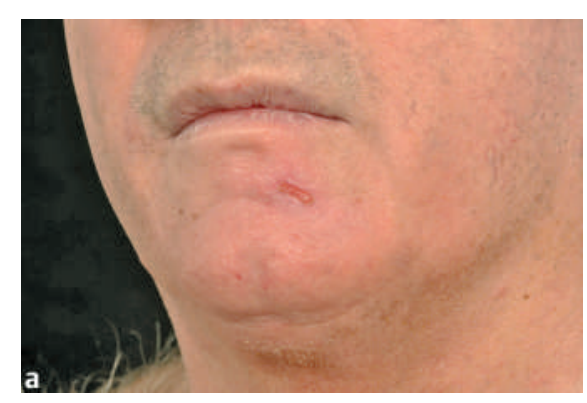

Abb. 2 a-c Befund nach kombinierter Therapie mit mikroverkapseltem Doxorubicin, PUVA und Interferon (a) Kinn, (b) Flanke (c) Stamm.
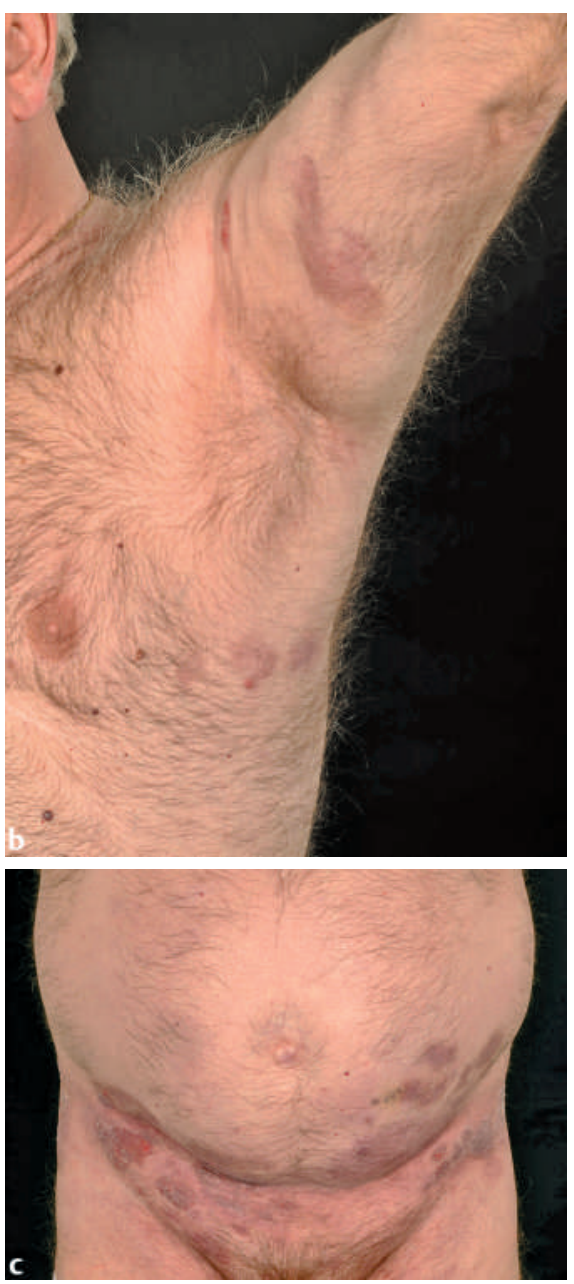

\section{Therapie und Verlauf}

- Chemotherapie mit PLD $20 \mathrm{mg} / \mathrm{m}^{2}$ KOF, 1. - 4. Zyklus in 3-wöchigen Abständen, 5. - 7. Zyklus in 6-8-wöchigen Abständen.

- Immuntherapie mit Interferon-alpha $3 \times 3$ Mio. s.c. I.E. pro Woche

- Creme-PUVA $3 \times$ wöchentlich.

- Symptomatisch zu Beginn Telfast (Fexofenadin) $180 \mathrm{mg}$ 1-0-0, Atarax (Hydroxyzin) 0-0-1.

- Topisch: initial Steroid Klasse I und desinfizierende Externa, zuletzt pflegende Lokaltherapie.

- Im Verlauf wurden insgesamt 8, teils atypische bzw. dysplastische Junktions- und Compoundnävi wegen der Creme-PUVA-Therapie operativ entfernt.

- Exstirpation des Nodus am Gesäß links (Histologie: organisiertes Hämatom).
Die Chemotherapie mit PLD wurde subjektiv sehr gut vertragen. Nach 4 Zyklen in 3-wöchigen Abständen traten leichte Thrombound Leukopenie sowie GPT-Erhöhung auf. Diese Werte stabilisierten sich mit Ausnahme der erhöhten Leberwerte im weiteren Behandlungsverlauf. EKG und Echokardiographie waren regelrecht. Nach der ersten Interferongabe hatte der Patient einmalig Schüttelfrost.

Unter o.g. Therapie waren die Knoten und Plaques kontinuierlich rückläufig außer an Kinn und Unterlippe. Letztere Areale wurden mit $4 \mathrm{MeV}$ Elektronenbestrahlung in Einzeldosen à $2 \mathrm{~Gy} 5 \times$ /Woche, Gesamtdosis $36 \mathrm{~Gy}$, erfolgreich behandelt (Abb.2a-c). Während dieser 4 Wochen wurden sowohl Chemo-, als auch Immuntherapie pausiert, um eine kombinierte Toxizität zu vermeiden. Der Juckreiz war nach 3 Zyklen komplett abgeklungen. Im Verlauf traten keine neuen Hautveränderungen auf. Viszerale Tumoren konnten radiologisch ausgeschlossen werden (s.o.).

\section{Diskussion}

Ziel der Behandlung der MF sind die Besserung des klinischen Erscheinungsbildes und die Prävention der Krankheitsprogression, von der letztendlich die Prognose abhängt.

Es gibt verschiedene topische und systemische Therapieoptionen, die allein oder in Kombination angewandt werden können. Therapieempfehlungen zur Behandlung der MF und MF-Sonderformen liegen in den Leitlinien der Deutschen Dermatologischen Gesellschaft vor. Standardisierte Therapieprotokolle besonders für die fortgeschrittenen Stadien der MF fehlen. Hier müssen bei der Entscheidung für eine bestimmte Behandlung Alter, Compliance des Patienten, Krankheitsstadium und -verlauf sowie bereits erfolgte Therapien berücksichtigt werden $[1,2]$.

In einer prospektiv randomisierten Studie wurden 103 Patienten mit MF untersucht und erhielten entweder Ganzkörperbehandlung mit schnellen Elektronen (GD 30 Gy) in Kombination mit einer Polychemotherapie oder verschiedene Lokaltherapien in Abhängigkeit des Krankheitsstadiums inklusive Phototherapie und Mechloretamin; in späteren Stadien konnte auch Radiotherapie oder Methotrexat eingesetzt werden. Die Response-Rate war bei der aggressiveren Behandlung mit Radiotherapie und Chemotherapie höher, aber mit ernsten Nebenwirkungen verbunden. Die Gesamtanalyse ergab keinen Einfluss auf das Gesamtüberleben. Da durch eine zu frühe aggressive Therapie das krankheitsfreie Intervall nicht verlängert wird, ist eine stadienabhängige Therapie wichtig [3].

Im fortgeschrittenen Stadium der MF kann die Lebensqualität durch schmerzhafte Ulzerationen und entstellende Tumoren stark eingeschränkt sein. Ohne Therapie haben die Patienten eine relativ kurze mittlere Lebenserwartung (ca. 40 Monate) $[4,5]$.

Doxorubicin ist ein Anthrazyklin mit zytostatischer Wirkung und wird in einigen Therapieprotokollen bei Non-Hodgkin-Lymphomen, z.B. im Rahmen des CHOP-Schemas, verwendet. Doxorubicin hat einen niedrigen therapeutischen Index, hämatologische Toxizität und dosisabhängige Nebenwirkungen sind häufig. Fast 
alle Patienten mit Dosierungen $>120 \mathrm{mg} / \mathrm{m}^{2}$ entwickeln starke Neutropenie. Gastrointestinale Nebenwirkungen, palmoplantare Erythrodysästhesie und reversible Alopezie sind häufig. Anthrazyklin-induzierte Kardiomyopathie kann zu Herzinsuffizienz führen. Zur Vorbeugung dieser irreversiblen Nebenwirkung darf die kumulative Lebenszeitdosis von Doxorubicin $450-550 \mathrm{mg} / \mathrm{m}^{2}$ nicht überschreiten.

In Liposomen eingeschlossene Medikamente bieten potenzielle Vorteile gegenüber den freien Medikamenten. So erreicht PLD eine 10fach höhere Wirkstoffkonzentration im Tumor als konventionelles Doxorubicin. Das Liposom verlängert die Halbwertszeit auf 74 Stunden. PLD wird durch das RES nicht erkannt. Seine Bioverfügbarkeit ist so verändert, dass sich PLD selektiv im Tumorgewebe anreichert, gesundes Gewebe wird geschont. Die Liposomen zirkulieren 2-3 Wochen nach der Infusion im Blutkreislauf, wobei Doxorubicin fast zu $100 \%$ in den Liposomen verkapselt bleibt. So wird eine kontinuierliche Freisetzung erreicht und kardiotoxische Spitzenspiegel vermieden [5].

PLD ist bei Patienten mit rezidivierender, therapieresistenter fortgeschrittener MF wirksam (Ansprechrate 88\%) und sicher in einer Dosis von $20 \mathrm{mg} / \mathrm{m}^{2}$ alle 4 Wochen. Eine klinische Besserung ist nach 3-monatiger Behandlung zu erwarten. Insgesamt werden nicht mehr als 8 Zyklen durchgeführt. Die unerwünschten Nebenwirkungen sind weniger schwer als bei anderen Chemotherapien. Kardiotoxizität und Nephrotoxizität sind geringer als bei konventionellem Doxorubicin. Ebenfalls werden durch den liposomalen Einschluss Übelkeit, Erbrechen und Alopezie signifikant reduziert. Hämatologische Nebenwirkungen sind häufig, jedoch meist milde und abhängig von Dosis und Behandlungsintervall. Schwere Stomatitis und Mukositis treten in weniger als $10 \%$ der Fälle auf und sind dosisabhängig. Nach wiederholter Gabe von PLD kann eine palmoplantare Erythrodysästhesie auftreten, die durch schmerzhafte Schwellungen mit fleckigen Rötungen der Palmae und Plantae gekennzeichnet ist. Darüber hinaus können Axillen, Leisten und Stellen, an denen die Kleidung eng sitzt betroffen sein. Die Patienten bemerken typischerweise zunächst ein unbestimmtes Taubheitsgefühl und Kribbeln, gefolgt von Ödemen $[5,6]$.
Die Kombination mit weiteren etablierten Therapien wurde bei dem Patienten eingesetzt, weil sich die Biologie der Tumorklone in schnell wachsenden Tumoren bei der MF von den Tumorzellen in Plaques und Patches bezüglich ihres Verhaltens unterscheiden. Die geringere Zellteilungsrate macht sie für Chemotherapien weniger empfänglich, daher sind intermittierende Therapie wie Interferon-alpha und PUVA besser geeignet.

Da es keine kurative Behandlung der MF gibt und aggressive Therapien trotz besserer Erfolge in der Anfangsphase die Prognose der Patienten nicht verbessern, ist die Behandlung mit PLD eine sinnvolle Alternative im fortgeschrittenen Stadium der MF; denn sie ist zum einen effektiv, zum anderen nebenwirkungsarm und weniger toxisch als andere Chemotherapien.

Der Einsatz von PLD in Kombination mit Interferon-alpha sollte weiterhin untersucht werden.

\section{Literatur}

${ }^{1}$ Anadolu RY, Birol A, Sanli H, Erdem C, Tursen U. Mycosis fungoides and Sezary syndrome:therapeutic approach and outcome in 113 patients. Int J Dermatol 2005; 44: 559-565

2 Lundin J, Osterborg A. Therapy for Mycosis fungoides. Curr Treat Options Oncol 2004; 5: 203 - 214

${ }^{3}$ Korting HC, Callies R, Reusch M, Schlaeger M, Sterry W. Dermatologische Qualitätssicherung, Leitlinien und Empfehlungen, 4. Auflage. Berlin: ABW Wissenschaftsverlag, 2005

${ }^{4}$ Tsatalas C, Martinis G, Magaritis D, Spanoudakis E, Kotsianidis I, Karpouzis A, Bourikas G. Long-term remission of recalcitrant tumour-stage mycosis fungoides following chemotherapy with pegylated liposomal doxorubicin. J Eur Acad Dermatol Venereol 2003; 17: 80 - 82

${ }^{5}$ Wollina U, Dummer R, Brockmeyer NH, Konrad H, Busch JO, Kaatz M, Knopf B, Koch HJ, Hauschild A. Multicenter Study of pegylated liposomal doxorubicin in patients with cutaneous T-cell lymphoma. Cancer 2003; 98: $993-1001$

${ }^{6}$ Di Lorenzo G, Di Trolio R, Delfino M, De Placido S. Pegylated liposomal doxorubicin in stage IVB mycosis fungoides. Br J Dermatol 2005; 153 : $183-185$ 